\title{
From crystallographic data to solution structure of photoreceptors: the case of AppA BLUF domain.
}

\author{
Shaima Hashem, Veronica Macaluso, Michele Nottoli, Filippo Lipparini, \\ Lorenzo Cupellini*广and Benedetta Mennucci*†
}

\begin{abstract}
Photoreceptor proteins bind a chromophore, which, upon light absorption, modifies its geometry or its interactions with the protein, finally inducing the structural change needed to switch the protein from an inactive to an active or signaling state. In the Blue Light-Using Flavin (BLUF) family of photoreceptors, the chromophore is a flavin and the changes have been connected with a rearrangement of the hydrogen bond network around it on the basis of spectroscopic changes measured for the dark-to-light conversion. However, the exact conformational change triggered by the photoexcitation is still elusive mainly because a clear consensus on the identity not only of the light activated state but also of the dark one has not been achieved. Here, for the first time, we present an integrated investigation that combines microsecond MD simulations starting from the two conflicting crystal structures available for the AppA BLUF domain with calculations of NMR, IR and UV-Vis spectra using a polarizable QM/MM approach. Thanks to such a combined analysis of the three different spectroscopic responses, a robust characterization of the structure of the dark state in solution is given together with the uncovering of important flaws of the most popular molecular mechanisms present in the literature for the dark-to-light activation.
\end{abstract}

\section{Introduction}

The availability of high-resolution crystallographic data has revolutionized our understanding of proteins. ${ }^{1}$ However, a crystal structure gives a single static representation, which is often not sufficient to explain how the protein really works. Moreover, in some cases, different structures can be solved for the same protein, thus making the structure-function prediction even more difficult. Such potential flaws become especially dangerous for proteins whose function is activated by a structural change. This is the case of photoreceptors, light-sensitive machines that regulate processes like growth, circadian rhythms and photomovement in bacteria, plants, and even some animals. Photoreceptor proteins bind a chromophore which, upon light absorption, undergoes a change in its internal geometry or in its interactions with some close protein residues. These local changes then propagate into the whole protein, finally inducing a conformational change, which switches the protein from an inactive, resting state to an active, signaling one. ${ }^{2-4}$
At variance with other proteins, what is compelling in photoreceptors is a detailed knowledge of the chromophore local environment, namely the type of residues, their 3D arrangement, the network of interactions among them, and their dynamics. Unfortunately, all this information cannot be easily and unequivocally obtained from the crystal structure. The latter in fact gives one single snapshot, which is not necessarily representative of the structures sampled by the system in solution. Moreover, relevant interactions can be missing or, conversely, artificial ones can be present due to the unavoidable limitations in the crystallographic resolution at the scale of atomic interactions. A possible strategy to overcome these problems is to supplement the crystallographic data with molecular dynamics (MD) simulations of the protein within its biological environment. ${ }^{5}$ However, for the MD results to be really useful, the conformational space should be properly sampled to avoid biases coming from the limitations of the initial crystal structures. An effective test to validate the completeness of the MD simulations is to integrate them with a quantum mechani- 
cal (QM) description, to predict spectroscopic properties that can be compared with experiments. This strategy requires to introduce a multiscale approach, where the QM description is combined with classical models such as the Molecular Mechanics (MM) force fields. In the resulting QM/MM model, three main aspects determine the quality of the description: the choice of the QM subsystem, the selected QM level and the coupling between the QM and the MM subsystems. ${ }^{6}$ In the case of photoreceptors, the first aspect is straightforward, as the QM description will necessarily include the chromophore and possibly a few close residues. Moreover, the dimensions of the chromophores present in photoreceptors and the need for a large sample size imposes the use of relatively inexpensive QM levels, such as Density Functional Theory (DFT), and its time-dependent (TDDFT) extension for simulating UV-Vis spectra. As regards the third aspect, the common strategy is to use an electrostatic embedding QM/MM formulation where the QM system "sees" the MM atoms as fixed point charges (or fixed multipoles). A more complete formulation of the QM-MM interactions is represented by a polarizable embedding, which explicitly accounts for mutual polarization between the QM and MM subsystems. The integration of polarizable QM/MM descriptions with extensive MD simulations has proven particularly effective in simulating spectra and, more in general, light-induced processes in complex biological systems. ${ }^{7}$

Here, this strategy is applied to a member of the Blue Light-Using Flavin (BLUF) family of photoreceptors present in bacteria. These proteins non-covalently bind a flavin chromophore, which upon absorption of blue light, initiates the cascade of structural changes leading to the active state. ${ }^{8-13}$ These changes have been connected with a rearrangement of the hydrogen bond network around the flavin on the basis of two spectroscopic signatures: a redshift in both the maximum of the UV-visible absorption spectrum and in the carbonyl infrared (IR) frequency of flavin. ${ }^{9,14}$ However, the exact conformational change triggered by the photoexcitation is still elusive. Strikingly, one of the reasons that prevents a complete and definitive characterization of the light-activation mechanism is the lack of a clear consensus on the identity not only of the light activated state of the protein but also of the initial (dark adapted) one. This problem is enhanced in AppA, a BLUF protein that controls photosynthesis in gene expression of the purple bacterium Rhodobacter sphaeroides. ${ }^{15}$ Two different crys- tallographic structures have been resolved for its resting state, which differ both in the composition and the arrangement of the protein residues of the flavin binding pocket (see Figure 1).

One structure shows a tryptophan (Trp104) in the binding pocket, and it is called $\operatorname{Trp}_{i n},{ }^{16}$ whereas, in the second, more recent structure, a methionine (Met106) replaces Trp104 in the binding pocket. This structure is denoted as Met $_{i n} .{ }^{17}$ Moreover, according to the $\operatorname{Trp}_{i n}$ structure, the Gln63 carbonyl group is oriented toward Trp104, while the Met $_{i n}$ structure suggests that the glutamine side chain is oriented with its carbonyl oxygen near the $\mathrm{OH}$ group of the tyrosine residue (Tyr21) and its amide nitrogen close to the flavin $\mathrm{O}_{4}$. These differences have significantly complicated the analysis due to the crucial role that all these residues have shown to play in the photoactivation. For example, the rotation ("flipping") or tautomerization of Gln63 have both been suggested as a possible change in the dark-to-light transformation ${ }^{13,16,18-25}$ and mutagenesis studies have shown the importance of Trp104 in the AppA activity. ${ }^{9,26-28}$

In the last fifteen years many computational studies have been performed to solve this controversy and identify the structures of both dark and light states of AppA. ${ }^{22,23,25,29-34}$ However, often the investigations have been based on crystal structures only or they have used excitation energies only. Even in the case of more extensive investigations, such as those combining the simulation of excitation energies and IR frequencies, a detailed analysis of the conformational space of the two alternative structures was not performed being the MD simulations limited to ten to few hundreds of nanoseconds. Here, for the first time, we present an integrated investigation that combines $20 \mu \mathrm{s}$ MD simulations starting from the two available structures of AppA with calculations of nuclear magnetic resonance (NMR), IR and UV-Vis spectra using a polarizable QM/MM approach. Moreover, the classical MD dynamics are here supplemented by polarizable QM/MM dynamics to investigate the IR frequencies beyond the standard harmonic approximation. Thanks to such a unique combination of advanced methods, a robust characterization of the structure of the dark state in solution is given together with the uncovering of important flaws of the most popular molecular mechanisms present in the literature for the dark-to-light activation. 

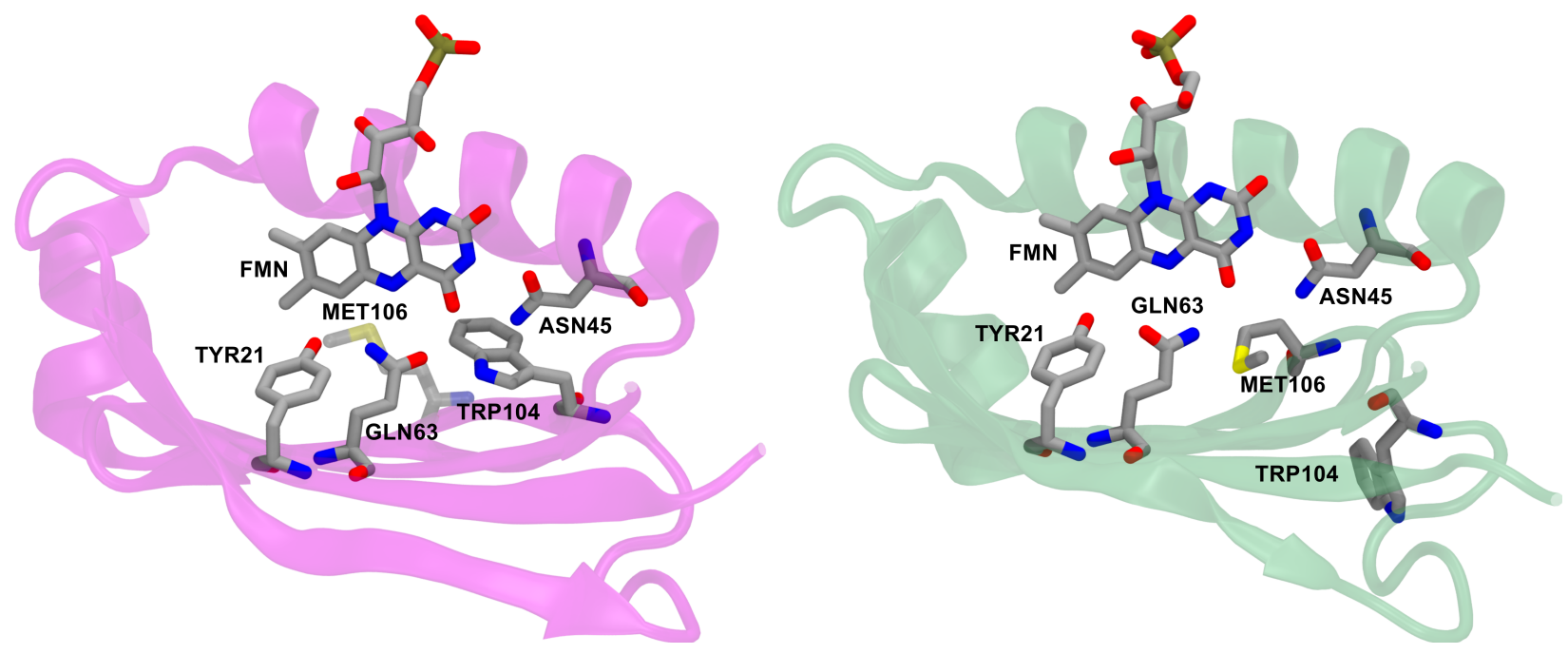

Figure 1 Flavin chromophore and the residues of the binding pocket in the two available crystal structures of AppA: Trp ${ }_{i n}{ }^{16}$ (left) and $\mathrm{Met}_{i n}{ }^{17}$ (right).

\section{Methods}

\subsection{Molecular Dynamics simulations}

The initial structures of AppA were extracted from the Protein Data Bank (PDB), describing Trp in (PDB ID:1YRX) ${ }^{16}$ and Met $_{\text {in }}$ (PDB ID:2IYG) ${ }^{17}$ conformations. In both structures the flavin chromophore is in the mononucleotide form (FMN). In addition to the main differences between the two structures reported in the Introduction and shown in Figure 1, we recall that in the Met $_{i n}$ structure, Cys20 is substituted by a serine. ${ }^{17}$

We applied our calculations on the monomer AppA instead of the naturally found dimer, so that our results can be easily compared to the previous computational studies. ${ }^{32}$ Additionally, it was found that the dimer interface significantly differs among the available crystal structures of AppA. ${ }^{16,17}$ We thus considered chain A, using residues 17 to 121 , in order to simulate the same sequence length for both structures. Hydrogen atoms were added using the LeAP module of AmberTools to refine the crystal structures. Protonation of the protein was set at $\mathrm{pH} 7$ using the $\mathrm{H}++$ web server. ${ }^{35}$ The positions of the hydrogen atoms were optimized using the Amber ff14SB force field. ${ }^{36}$

MD simulations were carried out using Amber18. ${ }^{37,38}$ Each system was soaked in a truncated octahedral box of TIP3P water molecules, ensuring a minimum $30 \AA$ separation between periodic images of the protein. The system was neutralized with counter-ions. The protein was described using Amber ff14SB force field, ${ }^{36}$ together with the general AMBER force field (GAFF), ${ }^{39}$ the parameters for the flavin mononucleotide molecule were obtained from a previous study. ${ }^{40}$ Periodic boundary conditions were applied. The Particle Mesh Ewald (PME) method was used to treat electrostatic interactions, using a cutoff of $10 \AA$. In all simulations, an integration time step of $2 \mathrm{fs}$ was employed together with the SHAKE algorithm to constrain all the bonds involving hydrogen atoms. The Langevin thermostat was used to control the temperature. Each system was minimized through 1000 steps of steepest descent followed by 1000 steps of conjugate gradient. A 50 ps NVT simulation was run increasing the temperature from 0 to $50 \mathrm{~K}$, followed by a $250 \mathrm{ps}$ of NPT simulation letting the system heat up to $300 \mathrm{~K}$. Positional restraints were applied on heavy atoms during the heating using a force constant of $4 \mathrm{kcal} / \mathrm{mol} / \AA^{2}$. $750 \mathrm{ps}$ of NPT simulation was then run to allow the box to equilibrate at $300 \mathrm{~K}$. NPT production simulations were performed for $2.5 \mu$ s for both $\operatorname{Trp}_{i n}$ and Met $_{i n}$ structures.

We initially performed two MD replicas of $2.5 \mu \mathrm{s}$ for each structure (MD1, MD2). As one of the the $\operatorname{Trp}_{i n}$ simulations manifested some instabilities (see below), we performed two additional control simulations (MD3 and MD4) with an extended equilibration for the loops in order to ensure the robustness of our findings. For the Met $_{\text {in }}$ simulations, we also performed two control simulations reverting the $\mathrm{C} 20 \mathrm{~S}$ mutation back to the original cysteine present in the AppA sequence (referred to in the following as "Met ${ }_{i n}-\mathrm{S} 20 \mathrm{C}$ " simulations. The total simulated time adds up to $20 \mu \mathrm{s}(8 \times 2.5 \mu \mathrm{s})$. A list of all 
simulations is reported in Table S1 in the ESI ${ }^{\dagger}$.

\section{$2.2 \mathrm{QM} / \mathrm{MM}$ optimizations}

Snapshots were extracted from the MD trajectories for each structure. These snapshots were taken from $2 \mu \mathrm{s}$ to $2.5 \mu \mathrm{s}$ of the production run every $5 \mathrm{~ns}$, yielding a total of 200 snapshots for each structure. All the snapshots were refined by $\mathrm{ONIOM}(\mathrm{QM}: \mathrm{MM})$ optimizations. ${ }^{41} \mathrm{In}$ these optimizations, only the FMN molecule was allowed to move, whereas the protein and solvent molecules were kept frozen. Only the isoalloxazine ring, including the C1' atom (Figure 4a), was treated at the QM level of theory (B3LYP-D3/6-31G(d) $)^{42}$, whereas the ribityl tail was treated at the MM level along with the rest of the system. All atoms within $20 \AA$ of the isoalloxazine ring, except the $\mathrm{Na}^{+}$and $\mathrm{Cl}^{-}$ions, were included in the $\mathrm{MM}$ part for the optimizations. The MM part was described with the same force field ${ }^{36,40}$ as in the MD simulations.

\section{$2.3 \quad$ Excited-state calculations}

The 200 selected snapshots were used for excited-state calculations performed using a polarizable QM/MM model $^{43,44}$ (from now on, QM/MMPol), which is based on the IDP formulation and describes the MM part as a set of point charges and isotropic polarizabilities from the pol12 AL Amber force field. ${ }^{45,46}$ The QM part consisted of the isoalloxazine ring of the FMN, including the C1' atom, and was treated at the $\omega \mathrm{B} 97 \mathrm{X}-\mathrm{D} / 6-31+\mathrm{G}(\mathrm{d})$ level. The protein, ions, and water molecules within $40 \AA$ of the chromophore were treated at the MMPol level. Although the long-range corrected $\omega \mathrm{B} 97 \mathrm{X}-\mathrm{D}$ gives a systematic blue shift with respect to experiment $(\sim 0.3 \mathrm{eV})$, we chose this functional in order to avoid artificial mixing of the first excited state given by standard hybrid functionals such as B3LYP, as done in our previous work on another flavoprotein. ${ }^{47}$

The homogeneous line shape of the flavin excitation was computed in the second-order cumulant expansion formalism. ${ }^{48}$ In this formalism the vibronic couplings with all the normal modes are encoded in the spectral density, namely

$$
J(\omega)=\pi \sum_{k} S_{k} \omega_{k}^{2}\left(\delta\left(\omega-\omega_{k}\right)-\delta\left(\omega+\omega_{k}\right)\right)
$$

where $S_{k}$ is the Huang-Rhys factor along mode $k$ with frequency $\omega_{k}$. The Huang-Rhys factors were calcu- lated by projecting the excited-state gradient onto the ground-state normal modes calculated at the B3LYPD3/6-31G(d) level, in the same ONIOM scheme described above. Homogeneous line shapes were computed on both $\operatorname{Trp}_{i n}$ and Met ${ }_{i n}$ crystal structures, but we found negligible differences between the two structures. Finally, the absorption spectra were computed by convoluting the homogeneous line shape with the inhomogeneous distribution of vertical excitation energies computed along the MD simulations. This strategy has recently proven effective in describing the absorption line shapes of flavoproteins. $^{47}$

\subsection{Chemical shift calculations}

For all of the optimized structures, we computed the chemical shifts for all protons of the FMN ring and the four most tightly interacting protein sidechains, as defined in our previous work. ${ }^{49}$ The environment effect on chemical shifts was treated at the same QM/MMPol approach used for the excited state calculations, where this time the QM part comprised the isoalloxazine ring and the side chains of the hydrogen-bonded residues (Gln63, Ty21 and Asn45) plus Trp104 and Met106, respectively for $\operatorname{Trp}_{i n}$ and $\mathrm{Met}_{\text {in }}$ structures. The QM part was described at the B3LYP/6-311+G(d,p) level, which was shown to well reproduce the much more expensive MP2/6-311G(d,p) calculations. ${ }^{49}$ The chemical shifts were averaged on either the $\operatorname{Trp}_{i n}$ or the $\operatorname{Met}_{i n}$ frames for comparison with experiments. Confidence intervals for the mean value were estimated by dividing the frames into contiguous blocks of 10-15 frames, and then using the block average to estimate error bars with the bootstrap method. This allows a more reliable error estimation that is not affected by correlations between consecutive frames.

\subsection{Polarizable QM/MM MD simula- tions and IR frequencies}

Polarizable $\mathrm{QM} / \mathrm{MM}$ MD simulations were performed starting from 10 frames extracted respectively from the $\operatorname{Trp}_{i n}$ and Met $_{i n}$ simulations. For each starting frame, a $10 \mathrm{ps} \mathrm{QM} / \mathrm{MM}$ MD was performed in the NVT ensemble with open boundary conditions. The QM/MMPol simulations were carried out using an interface ${ }^{50-52}$ between the molecular dynamics package Tinker ${ }^{53,54}$ and the development version of Gaussian. ${ }^{44,55}$ For these sim- 
ulations, the QM part consisted of the FMN isoalloxazine ring, which was treated at the $\omega$ B97X-D/6-31G(d) level. The MM part consisted of all atoms within $20 \AA$ of the QM part, and was described with the AMOEBA force field. ${ }^{56,57}$ Further details on the preparation of the $\mathrm{QM} / \mathrm{MMPol} \mathrm{MD}$ simulations are given in the $\mathrm{ESI}^{\dagger}$.

Vibrational frequencies were extracted from the last 5 ps of the QM/MM MD simulations. Power spectra were computed by Fourier transforming the autocorrelation function of bond lengths and bond angles, as well as their linear combinations. Since the normal modes are delocalized on the flavin ring, different modes contribute to the power spectrum of a single coordinate, and the power spectra show peaks at various frequencies. In order to better separate normal-mode frequencies, we resorted to a signal-processing technique called second-order blind identification (SOBI). ${ }^{58}$ Briefly, SOBI disentangles signals at different frequencies by performing a joint diagonalization of time-lagged autocorrelation matrices at various lag times. These autocorrelation matrices are here built on a basis of internal coordinates comprising all bond lengths and those bond angles that involve hydrogen atoms. The resulting linear combinations of internal coordinates showed power spectra that are well localized in frequency. More details on the frequency extraction are given in the ESI ${ }^{\dagger}$.

\section{Results}

\subsection{MD of the $\operatorname{Trp}_{i n}$ and Met Mn $_{i n}$ structures}

As detailed in the Methods section, various MD trajectories were run starting from both crystal structures (PDB IDs: $1 \mathrm{YRX}^{16}$ and $2 \mathrm{IYG}^{17}$ ) from now on $\operatorname{Trp}_{i n}$ and $\mathrm{Met}_{\text {in }}$ respectively.

We first examined the evolution of the backbone root mean square deviation (RMSD) of the protein core of $\operatorname{Trp}_{\text {in }}$ and $\mathrm{Met}_{i n}$ to their respective crystal structures, excluding the loop region (residues 95-103) and the last few residues of the C-terminal which are expected to be highly disordered. ${ }^{26}$ Two example RMSD plots are shown in Figure 2a. The $\mathrm{Met}_{i n}$ simulation is generally more stable along the whole trajectory compared to the $\operatorname{Trp}_{\text {in }}$ one. This is confirmed by the distribution of RMSD values (Figure S1), which are shifted to larger values for all $\operatorname{Trp}_{\text {in }}$ simulations, including the control Trp ${ }_{\text {in }}$ simulations with a modified equilibration protocol (MD3 and MD4). On the contrary, the $\mathrm{Met}_{i n}-\mathrm{S} 20 \mathrm{C}$ simulations are
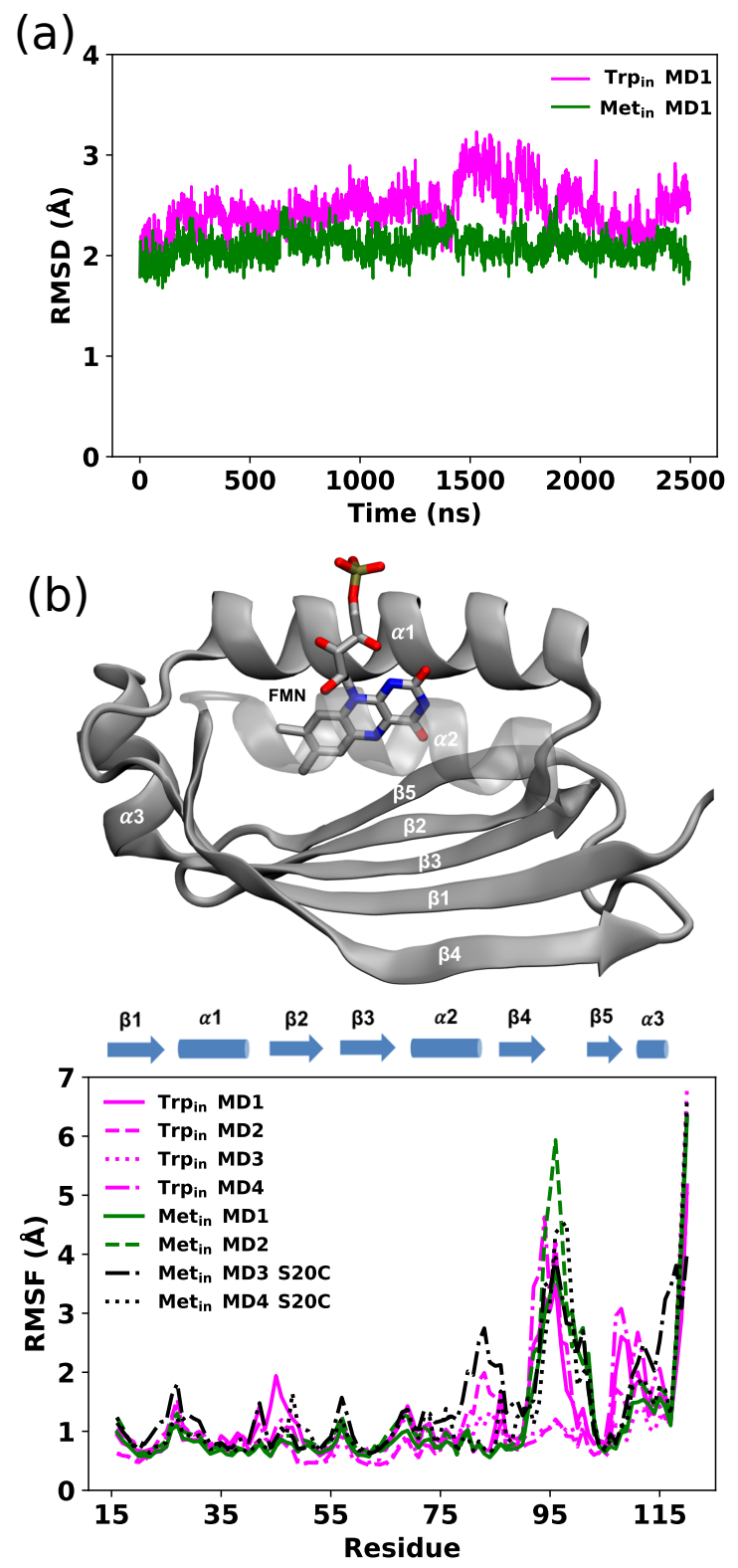

Figure 2 (a) Backbone RMSD along the $\operatorname{Trp}_{\text {in }}$ (magenta) and Met $_{\text {in }}$ (green) MD simulations. (b) Profiles of RMSF values for $\operatorname{Trp}_{i n}$ (magenta), Met ${ }_{i n}$ (green) and Met $_{i n}-\mathrm{S} 20 \mathrm{C}$ (black) MD simulations (lower panel). All replicas are reported. RMSF values were calculated on $\mathrm{C}^{\alpha}$ atoms coordinates of snapshots extracted from the production trajectory every 100 ps. The structure of Holo-AppA binding FMN, showing the different subdomains is also reported (upper panel).

more dynamic and reach higher RMSD values.

We evaluated the overall dynamics of both structures as the root mean square fluctuation (RMSF) on the $\mathrm{C}^{\alpha}$ atoms (Figure $2 \mathrm{~b}$ ). In addition to the flexible regions of the loop and the C-terminal, all $\operatorname{Trp}_{i n}$ simulations exhibit large fluctuations especially in the $\alpha 1$ helix, $\beta 5$ strand and the C-terminal helix ( $\alpha 3$ helix) compared to 
(a)
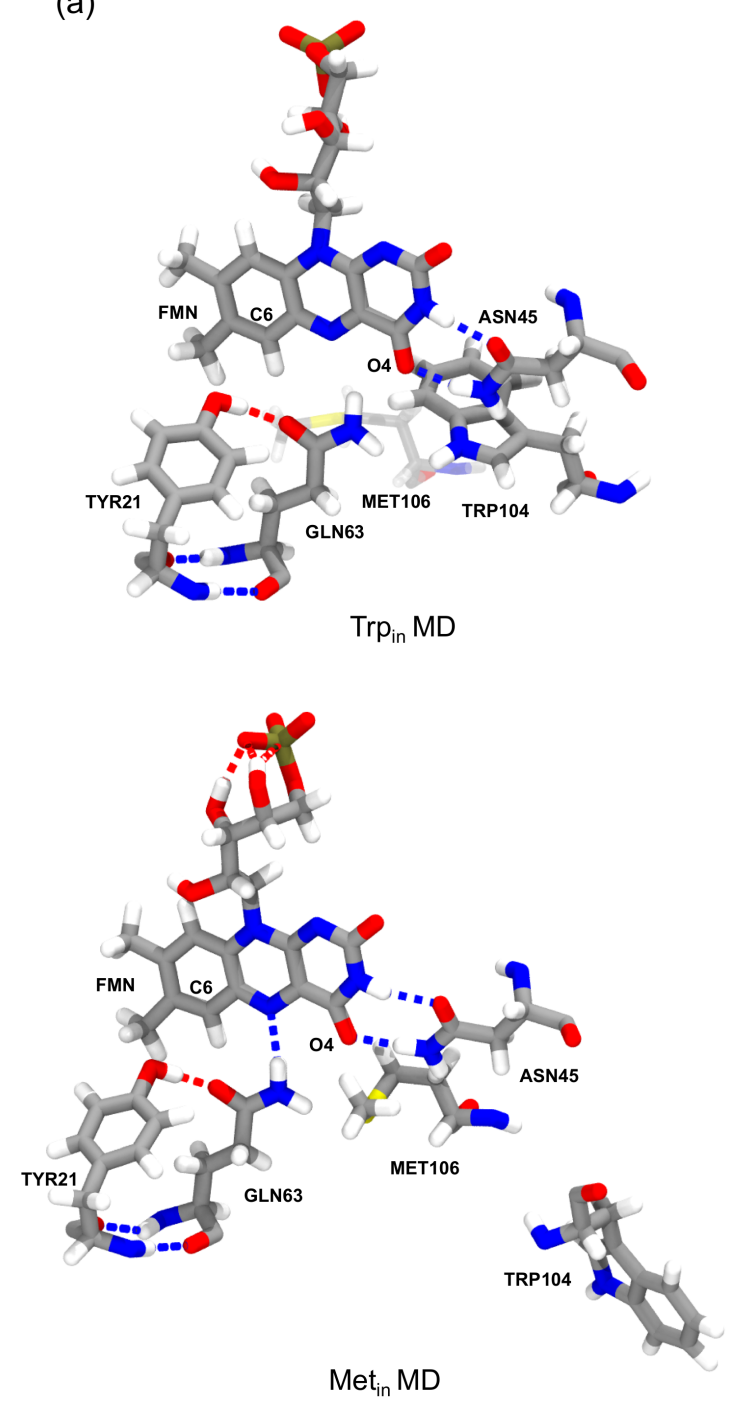

(b)

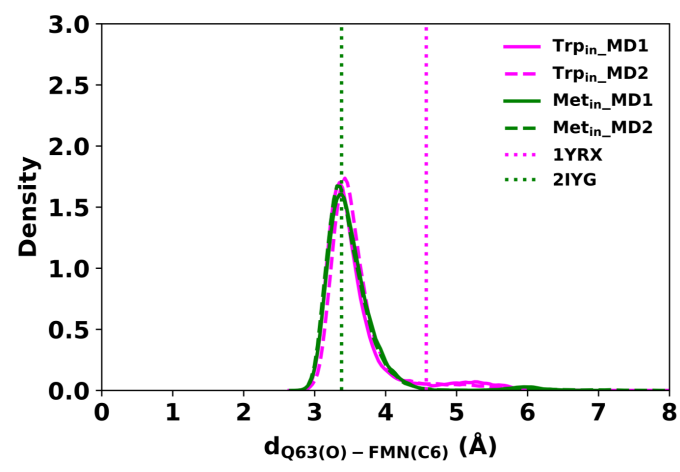

(c)

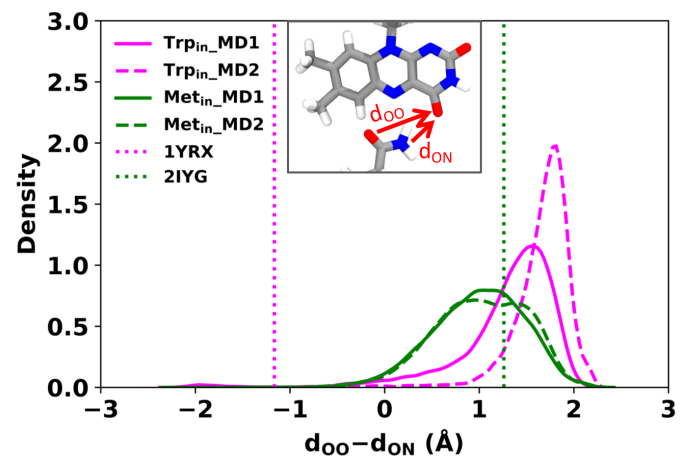

(d)

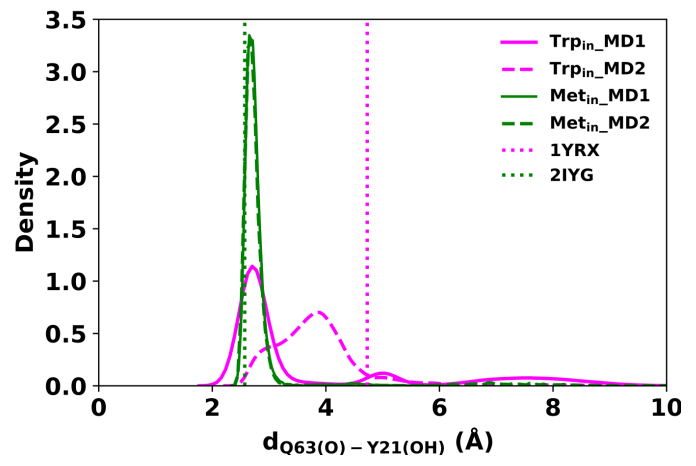

Figure 3 (a) FMN binding pocket in a representative snapshot of the Trpin (left) and $\operatorname{Met}_{i n}$ (right) MD simulations. (bd) Distributions of the distances detecting Gln63 motion along the $\operatorname{Trp}_{i n}$ (magenta) and Met $_{i n}$ (green) MD simulations. (b) Distribution of the distance between the oxygen of Gln63 and C6 of FMN. (c) Distribution of the difference between FMN $\left(\mathrm{O}_{4}\right)$ Gln63(O) distance denoted as $d_{O O}$ and $\mathrm{FMN}\left(\mathrm{O}_{4}\right)-\mathrm{Gln} 63(\mathrm{~N})$ distance denoted as $d_{O N}$. (d) Distribution of the distance between the oxygen of Gln63 and the hydroxyl group of Tyr21. The distances obtained from the Trp ${ }_{i n}$ (1YRX) (magenta) and Met ${ }_{i n}$ (2IYG) (green) crystal structures are represented as dotted lines.

the $\mathrm{Met}_{i n}$ ones. Only the two $\mathrm{Met}_{i n}-\mathrm{S} 20 \mathrm{C}$ simulations display large motions similar to the $\operatorname{Trp}_{\text {in }}$ ones, or even larger. These results suggest that the C20S mutation performed in ref. 17 has a stabilizing effect on the Met $_{i n}$ conformation of AppA.

In all $\operatorname{Trp}_{\text {in }}$ simulations, we observed relevant changes in the flavin binding pocket. The side chain of Gln63 adopts a conformation analogous to the Met ${ }_{i n}$ structure, and opposite to the crystal conformation depicted in Figure 1. The hydrogen bonding pattern thus changes, and becomes more similar to the Met $_{i n}$ structure (see Figure 3a). This new conformation of Gln63 can be quantified by the distance distributions shown in Figure 3b. In both $\operatorname{Trp}_{i n}$ and $\mathrm{Met}_{\text {in }}$ simulations, the oxygen of Gln63 remains close to the $\mathrm{C} 6$ atom of the flavin, at a distance comparable to the $\mathrm{Met}_{i n}$ structure. The same conclusion can be taken by looking at the difference between $\operatorname{FMN}\left(\mathrm{O}_{4}\right)-\mathrm{Gln} 63(\mathrm{O})$ and $\mathrm{FMN}\left(\mathrm{O}_{4}\right)-\mathrm{Gln} 63(\mathrm{~N})$ distances $\left(d_{O O}-d_{O N}\right)$ in Figure $3 \mathrm{c}$, which shows that the Gln63 amide nitrogen is closer to the flavin $\mathrm{O}_{4}$ atom than the 
amide oxygen. With this conformational change, Gln63 establishes an interaction with Tyr21 similar to that observed in $\mathrm{Met}_{i n}$, with Gln63(O) and Tyr21(OH) approaching a H-bonding distance (Figure 3d). However, this interaction is more dynamic than in the Met ${ }_{i n}$ structure, resulting in broader distributions. Control Trp ${ }_{i n}$ replicas performed after equilibration of the mobile loops (MD3 and MD4) all showed the same characteristics (Figure S2), with the Gln63 side chain oriented as in the Met $_{i n}$ structure. These results strongly suggest that the orientation of Gln63 proposed by Anderson et al. ${ }^{16}$ (Figure 1) is not stable in the $\mu$ s time scale. However, while rotation of Gln63 was observed already at the beginning of $\operatorname{Trp}_{i n}$ simulations, the conformation shown in Figure 3a only stabilized after 200-900 ns (see Figure S5 in the $\mathrm{ESI}^{\dagger}$ ), depending on the replica. This fact underlines the importance of long-scale MD simulations to correctly identify the most stable conformation of the flavin binding pocket.

To better identify and understand the structural changes occurring in the binding pocket, hydrogen bond analysis was performed among the main key residues surrounding the flavin and between these residues and the flavin. These hydrogen bonds were previously proposed to have an important role in the AppA photocycle. ${ }^{59,60}$ The probability of occurrence of these hydrogen bonds according to the time of the simulations is reported in Table S2. The hydrogen bond between $\mathrm{Gln} 63(\mathrm{O})$ and Tyr21(OH) was observed during all the simulations with higher probability in the Met ${ }_{i n}$ simulations (with an average of $85 \%$ ) than the $\operatorname{Trp}_{\text {in }}$ ones (with an average of $55 \%$ ). By contrast, the Gln63(NH) $\cdots$ Tyr21(O) hydrogen bond has negligible occupancy in the $\operatorname{Trp}_{i n}$ simulations (5\%), and it was never observed in the Met ${ }_{i n}$ simulations.

In the $\operatorname{Trp}_{i n}$ simulations, the hydrogen bonds between Asn 45 and FMN are temporarily lost in two of the four replicas that were performed (Figure S3), and are only recovered after $\sim 2 \mu \mathrm{s}$. This loss of interaction mirrors a change in the tertiary structure of the protein, as seen from the increased RMSD to the crystal structure (Figure 2b). Asn45(NH) binds to $\operatorname{FMN}\left(\mathrm{O}_{4}\right)$ with average probabilities of $76 \%$ and $67 \%$ for $\operatorname{Trp}_{i n}$ and Met ${ }_{i n}$ simulations, respectively. Instead, the hydrogen bond between Asn45(O) and $\mathrm{FMN}\left(\mathrm{N}_{3} \mathrm{H}\right)$ is retained along the whole Met $_{\text {in }}$ trajectories (90\%) (Figure S4) while it is more frequently lost along the $\operatorname{Trp}_{i n}$ trajectories (63\%) (Figure S3).

Notwithstanding the conformational change of Gln63, and the consequent loss of the Gln63 $\cdots$ Trp104 hydrogen bond, we do not observe substantial changes in the Trp104 position along our simulations. The flavin binding pocket thus remains stable in the $\operatorname{Trp}_{i n}$ conformation. Analogously, in the Met ${ }_{i n}$ simulation, Met106 retains its position inside the binding pocket for all the simulations.

From this analysis, we conclude that both $\operatorname{Trp}_{\text {in }}$ and Met $_{\text {in }}$ conformations are local free-energy minima, i.e. metastable states in the microsecond time scale and any conformational rearrangement between these two structures requires a significantly longer time. ${ }^{23}$

\subsection{NMR Chemical shifts}

The structures obtained from the MD simulations have been used for the QM/MMPol calculation of chemical shifts. For this investigation all the snapshots extracted from the MD simulations were refined by geometry optimizations of the flavin molecule within the frozen environment made of the protein and solvent molecules (see the Methods section for details). To compare with experiments, we present the data relative to the protons of the isoalloxazine ring and those of the common surrounding residues in the flavin binding pocket, i.e. Tyr21, Asn45, and Gln63 (See Figure 4a).

Figure $4 \mathrm{~b}$ shows the calculated $\operatorname{Trp}_{i n} / \operatorname{Met}_{i n}$ chemical shifts compared to experiments. ${ }^{59}$ The agreement is generally good for both structures, and in all cases calculations reproduce the highly de-shielded $\mathrm{H} 3$ proton. However, the coefficient of determination $\left(R^{2}\right)$ is better for the Met $_{i n}$ structure than for the $\operatorname{Trp}_{i n}$ structure. The largest discrepancy with respect to experiments is found for the amide HD22 of Asn45 for the $\operatorname{Trp}_{\text {in }}$ simulations (Figure 4b, left): the calculated chemical shift of this proton which is close close to the aromatic ring of Trp104 is in fact much lower than the measured one. On the Met $_{\text {in }}$ structure, instead, our calculations predict a chemical shift much closer to the experiment, and similar to the corresponding Gln63 amide proton.

To have a more detailed understanding of these findigs in Figure 4d we report the distributions of amide chemical shifts calculated for Asn 45 and Gln63 residues. The plots show similarities between Met $_{i n}$ and Trp ${ }_{i n}$ structures, except for the aforementioned HD22 atom of Asn45, which has a completely different distribution in the two structures. In $\operatorname{Trp}_{i n}$, the HD22 chemical shift has a broader distribution, centered on lower chemical shift values.

We hypothesize that Trp104 might be directly respon- 

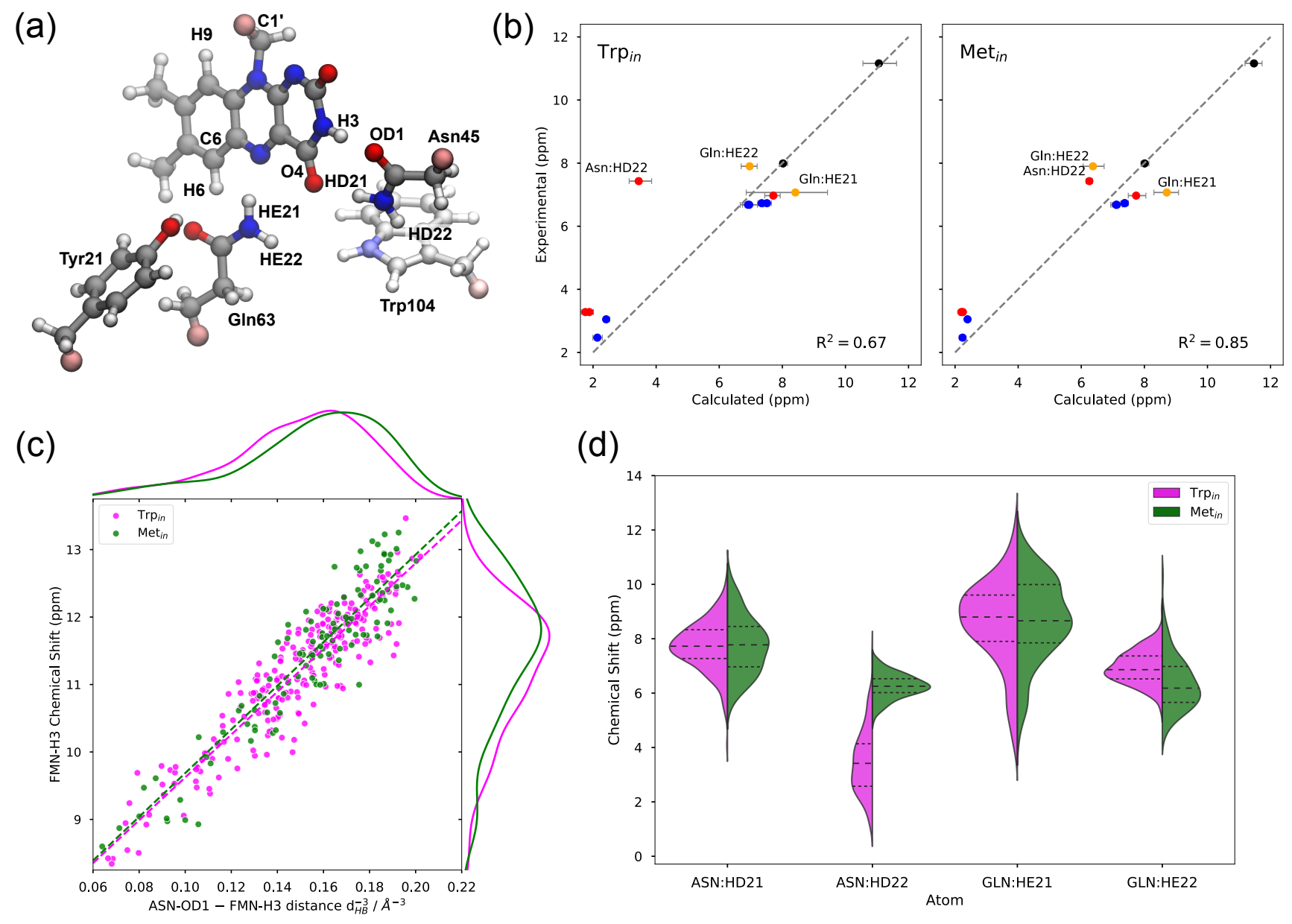

Figure 4 (a) Representation of the QM system considered for the QM/MMPol calculations, from a representative frame of the $\operatorname{Trp}_{i n}$ simulation. In the Met ${ }_{i n}$ structure, Met106 is included in place of Trp104. Link atoms are shown in pink color. (b) Comparison between calculated and experimental chemical shifts of Trp in $_{\text {(left) and Met }}$ in (right), including the protons of FMN (black), Tyr21 (blue), Asn45 (red), and Gln63 (yellow). Points with the largest deviations are labeled. Error bars represent bootstrapped 95\% confidence intervals. (c) Dependence of the $\mathrm{H}_{3}$ chemical shift on the inverse third power of the H-bond distance $\left(d_{H B}^{-3}\right)$, between the Asn45 OD1 atom and the FMN $\mathrm{H}_{3}$ atom. The dashed line represents a fit $\delta=\delta^{0}+a \cdot d_{H B}^{-3}$, where a different slope $a$ is allowed for $\operatorname{Trp}_{i n}$ (magenta) and Met ${ }_{i n}$ (green). The marginal distributions for $d_{H B}^{-3}$ (top) and for the chemical shift (right) are calculated with Gaussian kernel density estimation. (d) Distribution of amide protons chemical shifts of Asn45 and Gln63 for both $\operatorname{Trp}_{i n}$ (magenta) and Met $_{i n}$ (green) structures.

sible for the unusual shielding of the Asn45 HD22 proton, owing to the shielding cone generated by the aromatic indole ring of the tryptophan. In fact, in the Trp ${ }_{i n}$ structure, the Asn 45 amide group lies above the center of the pyrrolic ring of Trp104 (Figure 4a), whereas in the Met $_{\text {in }}$ structure Trp104 is far from the pocket. In order to assess the direct role of the tryptophan, we recomputed the chemical shifts on the Trpin frames after moving Trp104 to the MM part. Comparing the two calculations (Figure S5a), it becomes clear that Trp104 has a direct, quantum mechanical, shielding effect on HD22 in most of the frames, whereas the other amide protons are only marginally affected. As a consequence, the shield- ing effect of Trp104 modifies the HD22 chemical shift distribution both in the position and in the broadening (Figure S5b). Notably, the anomalous shielding of the Asn 45 amide proton was also previously found in the calculations on the $\operatorname{Trp}_{\text {in }}$ crystal structure 1YRX, at both B3LYP and MP2 levels of theory, ${ }^{49}$ indicating that this result is not affected by our sampling of the $\operatorname{Trp}_{\text {in }}$ structure, nor by the level of theory used in this work.

Protons involved in hydrogen bonding interactions, or close to polar residues, are the most sensitive to changes in the local sidechain arrangement. Among these, flavin $\mathrm{H}_{3}$ was shown to be sensitive to the dark-to-light transition of AppA, giving the largest chemical shift change 
$(+\sim 0.6 \mathrm{ppm}) .{ }^{20}$ Our calculations predict very similar $\mathrm{H}_{3}$ chemical shift (within error) in $\mathrm{Met}_{i n}$ and $\operatorname{Trp}_{i n}$ structures, thus suggesting caution in connecting the darkto-light change (or vice versa) to an exchange between tryptophan and methionine inside the binding pocket.

In order to quantify the sensitivity of the $\mathrm{H}_{3}$ chemical shift to the H-bond pattern, we sought a relationship with the H-bonding distance to Asn45(OD1) $\left(d_{H B}\right)$. Our results show that the $\mathrm{H}$-bond effect on this chemical shift is proportional to the inverse third power of $d_{H B}$ (Figure $4 \mathrm{c}$ ), and increases to 11-12 ppm when the Asn45 oxygen is at hydrogen bonding distance. Notably, the same trend can be observed for both Met $_{i n}$ and $\operatorname{Trp}_{\text {in }}$ structures, with a minimal difference in the slope. The distribution of $d_{H B}^{-3}$ (Figure 4c, top) is slightly different for the two structures, with Met $_{\text {in }}$ showing closer H-bonding interactions, and is reflected in the distribution of chemical shifts (Figure 4c, right). However, the difference between the two means $(\sim 0.3 \mathrm{ppm})$ is smaller than the statistical error, and it is too small to explain the observed $\sim 0.6$ ppm dark-to-light change.

Looking at the relationship of Figure 4c, it is clear that structures with a tight hydrogen bond $\left(d_{H B}<1.85 \AA\right)$ give rise to $\mathrm{H}_{3}$ chemical shifts around $12 \mathrm{ppm}$, i.e. compatible with the light-induced state of AppA. ${ }^{20}$ We can thus hypothesize that the light-induced AppA state will be characterized by a strong hydrogen bond with smaller fluctuations compared to what observed in our MDs.

\subsection{IR and UV-Vis signatures}

As reported in the Introduction, the two spectroscopic signatures used to characterize the structural changes between the dark (inactive) and the light-activated forms of AppA are the redshifts observed in the flavin absorption maxima $(\sim 10 \mathrm{~nm}$, e.g. $\sim 0.07 \mathrm{eV})$ and in the IR frequency of its $\mathrm{C}_{4}=\mathrm{O}_{4}$ stretching mode $\left(20 \mathrm{~cm}^{-1}\right) .{ }^{8,61} \mathrm{It}$ thus becomes interesting to investigate if the differences previously found in the chemical shifts of the Met ${ }_{i n}$ and $\operatorname{Trp}_{\text {in }}$ configurations have any connection with changes in the absorption maxima and the carbonyl frequencies which can finally be used to explain the molecular origin of the dark-to-light signatures.

To simulate the IR frequencies of the carbonyl stretching modes, we randomly extracted a subset of ten $\operatorname{Trp}_{i n}$ and ten Met ${ }_{i n}$ configurations and used them in combination with normal-mode harmonic calculations. The obtained values however showed an unexpected problem: for almost all the configurations a the $\mathrm{C}_{2}=\mathrm{O}_{2}$ stretching frequency is blue-shifted with respect to the $\mathrm{C}_{4}=\mathrm{O}_{4}$ one, which is in contradiction with experiments ${ }^{27,62}$. We note that in the isolated flavin, the same QM calculations indicate a $\mathrm{C}_{2}=\mathrm{O}_{2}$ frequency slightly redshifted with respect to the the other carbonyl. Therefore, the most probable reason for the observed wrong behavior is the way the harmonic calculations account for the environment. Here, in fact, the frequency differences are due to the different local environment of the two carbonyl groups both in terms of the number and the type of protein residues surrounding them, their orientations and the eventual presence of water molecules at hydrogen-bonding distances with the two carbonyl oxygens. To investigate this issue, we checked possible correlations between the frequencies of the two modes and the distances with potential hydrogen bonding donors. From the analysis reported in the previous sections, the most interesting candidate for $\mathrm{O}_{4}$ is Gln63. The results of this investigation are shown in Figure $\mathrm{S} 7$ of the $\mathrm{ESI}^{\dagger}$ where we report a correlation between the $\mathrm{C}_{4}=\mathrm{O}_{4}$ frequency and the $\mathrm{O}_{4}-\mathrm{Gln} 63(\mathrm{H})$ distance in the different $\operatorname{Trp}_{i n}$ and Met ${ }_{i n}$ optimized structures. As it can be seen, a clear correlation is present for Met ${ }_{i n}$, for which a shorter distance corresponds to a smaller frequency as expected by the destabilization induced by the H-bond on the carbonyl double bond. On the contrary, the $\operatorname{Trp}_{\text {in }}$ structures do not show any clear correlation with such a distance, even if the sampled H-bond distances are similar to those found in Met $_{i n}$. Moving to the analysis of $\mathrm{C}_{2}=\mathrm{O}_{2}$, the only possible $\mathrm{H}$-bond donor is represented by water molecules. In all the selected frames, only few water molecules are present at H-bond distances but also in those cases the $\mathrm{C}_{2}=\mathrm{O}_{2}$ frequency is higher than the one localized on the other carbonyl.

This analysis clearly shows the limitations of harmonic calculations associated to optimized structures in the presence of an environment that can establish very dynamic H-bond interactions. To overcome these important limitations, we therefore resorted to a dynamical approach based on polarizable QM/MM MDs. We used the same $10+10$ configurations used for the harmonic calculations and we ran $10 \mathrm{ps} \mathrm{QM} / \mathrm{MM}$ MDs starting from each of these structures (see Methods section for details).

The $\mathrm{C}=\mathrm{O}$ mode frequencies were computed from the power spectra of linear combinations of internal coordinates. These linear combinations were determined by the SOBI signal-processing analysis (see Methods) and allowed to obtain power spectra well localized in fre- 
(a)

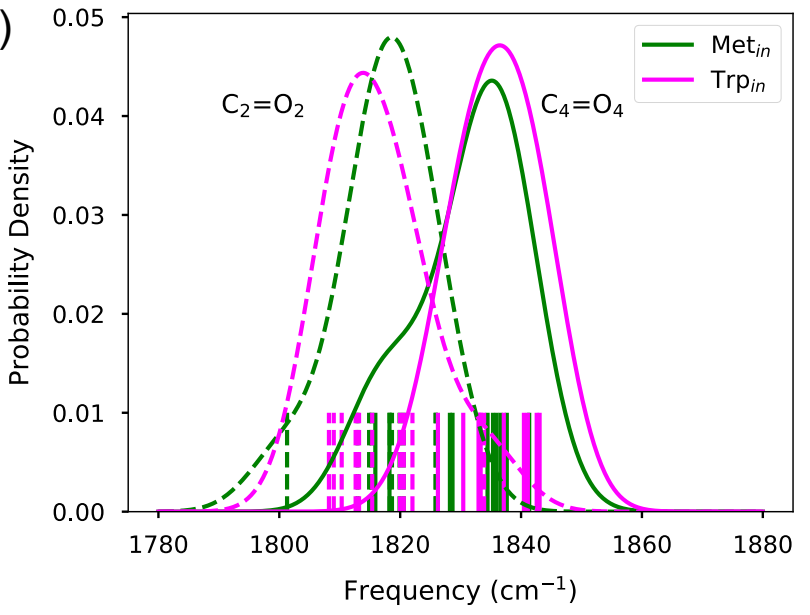

(c)

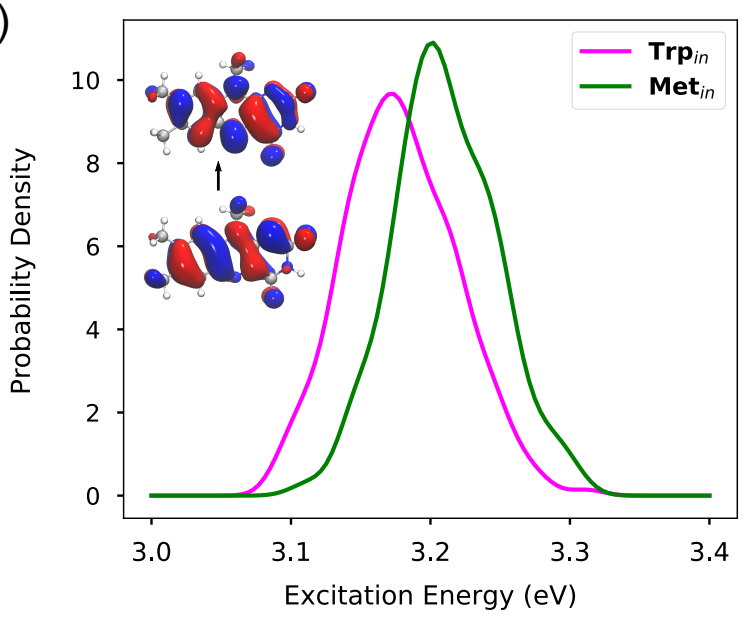

(b)

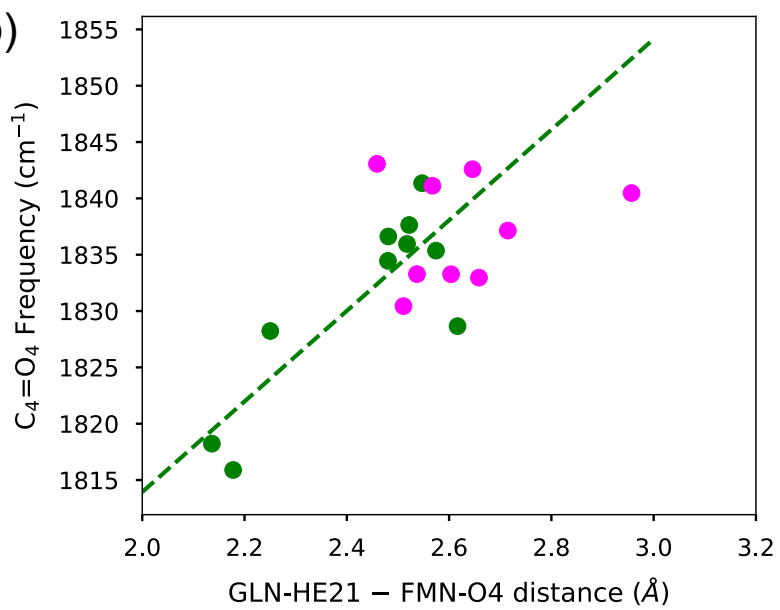

(d)

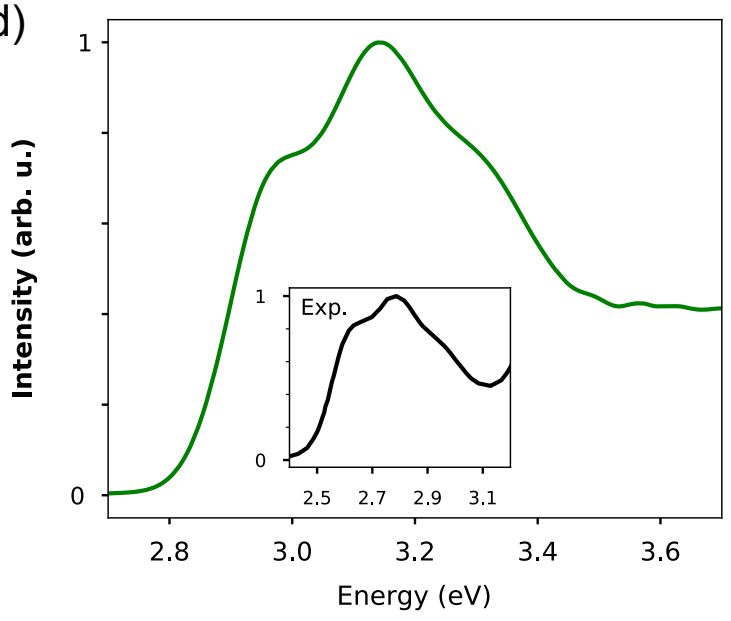

Figure 5 (a) Distribution of $\mathrm{C}=\mathrm{O}$ frequencies extracted from $10 \mathrm{Trp}_{\text {in }}$ and $10 \mathrm{Met}_{\text {in }}$ MDs. Individual frequencies are shown as vertical bars. The dotted and continuous lines represent the frequencies for $\mathrm{C}_{2}=\mathrm{O}_{2}$ and $\mathrm{C}_{4}=\mathrm{O}_{4}$ for Trp $\mathrm{T}_{\text {in }}$ (magenta) and Met min $_{\text {a }}$ (green) simulations, respectively. (b) Correlation between extracted $\mathrm{C}_{4}=\mathrm{O}_{4}$ frequencies and hydrogen bond distance from Gln63

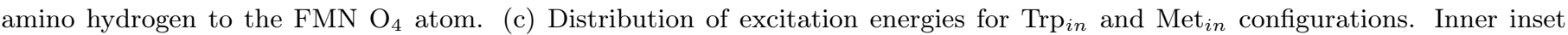
shows the electron-hole NTOs. (d) Absorption spectra calculated on Met ${ }_{i n}$ configurations. Inner inset shows the experimental absorption spectrum in black. ${ }^{61}$ The spectral intensities were normalized in order to have a maximum of 1 .

quency. As seen in Figure 5a, these frequencies are now in the same order as the experiments, with the $\mathrm{C}_{4}=\mathrm{O}_{4}$ frequency being $\sim 20 \mathrm{~cm}^{-1}$ higher than the $\mathrm{C}_{2}=\mathrm{O}_{2}$ indicated a more realistic description of environment effects.

Contrary to what observed in the harmonic calculations, in the $\mathrm{MD}$ trajectories, the $\mathrm{C}_{2}=\mathrm{O}_{2}$ group is always hydrogen bonded to at least one water molecule, and because of that it is difficult to assess the effect of such interaction on the frequencies. However, the $\mathrm{C}_{4}=\mathrm{O}_{4}$ experiences different situations within our trajectory samples. As before, also here, we have investigated the relationship between the $\mathrm{C}_{4}=\mathrm{O}_{4}$ frequency and the hydrogen bond with Gln63, through a correlation plot with the $\mathrm{O}_{4}-\mathrm{Gln}(\mathrm{H})$ distance (see Figure $5 \mathrm{~b}$ ). From the plot, it is evident that in the Met $_{i n}$ configurations the hydrogen bond well explains the value of the carbonyl frequency, and a clear correlation with the $\mathrm{Gln}(\mathrm{H})-\mathrm{O}_{4}$ distance is found. Moreover, we see that the distances explored in the $\mathrm{QM} / \mathrm{MM}$ dynamic trajectories correspond to a variability of the order of $30 \mathrm{~cm}^{-1}$ in frequencies, i.e. even more than what seen experimentally in the dark-to-light transition. This suggests that such a signature alone cannot be used to safely distinguish between potential dark or light-activated forms. On the other hand, in Trpin, the range of explored of $\mathrm{Gln}(\mathrm{H})-\mathrm{O}_{4}$ distances is much smaller and mostly concentrated in the large-value side of the plot. This indicates that the hydrogen bond is generally looser than in Met $_{i n}$. This behaviour can be traced 
back to the interaction of Gln63 with Tyr21. Indeed, in the Met $_{\text {in }}$ QM/MM MDs, Tyr21 always presents a tight interaction with the amide oxygen of Gln63. This interaction is much looser in the $\operatorname{Trp}_{\text {in }}$ configurations (Figure S8 in the $\mathrm{ESI}^{\dagger}$ ). In some $\operatorname{Trp}_{i n}$ trajectories, Tyr21 is very far from Gln63 but even in those trajectories where a hydrogen bond is possible, the interaction is much looser than in the $\mathrm{Met}_{\text {in }} \mathrm{QM} / \mathrm{MM}$ MDs. Even though the QM/MM MDs necessarily represent a limited sampling, they are completely in line with the classical MD results of Figure $3 \mathrm{c}$, which show a tighter interaction between Gln63 and Tyr21 for the Met ${ }_{i n}$ simulations.

To complete the investigation of the dark-to-light spectroscopic signatures, we calculated the excitation energies of Met $_{i n}$ and $\operatorname{Trp}_{i n}$ configurations using the computational protocol described in the Methods Section. The results, reported in Figure $5 \mathrm{c}$ in terms of distributions for the two sets of structures, show a rather unexpected behavior. As it can be seen from the NTOs depicted in the inset of $5 \mathrm{c}$, the $\pi-\pi^{*}$ excitation involves a large part of the molecule but still a weak transfer of electron density is visible between the two carbonyls. It is thus expected that H-bonds on those groups will induce a red-shift. Here, however, what we observe is that the $\operatorname{Trp}_{\text {in }}$ configurations which have shown much looser H-bond interactions at $\mathrm{O}_{4}$, present an excitation energy distribution which is red-shifted by $\sim 0.03 \mathrm{eV}$ with respect to Met $_{i n}$. To better understand this finding, we have isolated the effects that the protein has on the geometry of flavin, and indirectly on its excitation energy. We have thus recalculated the flavin excitation energies removing all the MMPol sites but still keeping the geometry as obtained in the original configurations. This analysis, which has been repeated for both $\mathrm{Met}_{i n}$ and $\operatorname{Trp}_{\text {in }}$ configurations, clearly shows that indeed the observed red-shift of $\operatorname{Trp}_{\text {in }}$ is due to the different geometrical constraints imposed on the flavin by the different local environment.

This result indicates that a very careful analysis has to be done when using calculated shifts on excitation energies to search for the correct dark and light structures, and that simulations of multiple spectroscopies have to be combined together in order to reach a physically sound picture. Here, in fact, the structures that have shown the worst agreement with experiments for both NMR and IR data, if incorrectly used as candidates for the dark state, would introduce an artificial red-shift in the absorption energy, which could invalidate the entire analysis.

Finally, the Met ${ }_{i n}$ structures have been used to cal- culate the lineshape using the approach described in the Methods Section. The resulting spectrum is reported in Figure 5d where it is also compared with the experimental one. Our calculations well reproduce the partially resolved vibronic structure, which present a main $0 \rightarrow 1$ peak and two shoulders corresponding to the fundamental and $0 \rightarrow 2$ transitions. The vibronic lineshape arises from the coupling of the excitation with several flavin ring modes; analysis of the Huang-Rhys factors (see Methods section) shows that both $\mathrm{C}=\mathrm{C}$ and $\mathrm{C}-\mathrm{C}$ ring modes have significant vibronic coupling. Comparison with our previous work, ${ }^{47}$ where a slightly different lineshape was obtained for flavin absorption in mutant LOV2 flavoproteins, suggests that the lineshape can be tuned by the interaction with the protein.

\section{Discussion}

The microsecond scale simulations performed in this work have provided a new picture of the AppA structure in solution. Most strikingly, our results strongly indicate that the conformation adopted by Gln63 is essentially the same for $\operatorname{Trp}_{\text {in }}$ and Met $_{\text {in }}$ binding pockets (Figure 3). Irrespective of the position of Trp104, in fact, Gln63 is preferentially oriented with the oxygen away from the flavin $\mathrm{O}_{4}$ atom. As a consequence, the hydrogen bond pattern in the AppA pocket is very different from what inferred by Anderson et al. ${ }^{16}$. Gln63 acts as a H-bond acceptor towards the hydroxyl group of Tyr21, in the Trp ${ }_{i n}$ as in the Met $_{\text {in }}$ structure, whereas the Gln63(O) $\cdots \operatorname{Trp} 104(\mathrm{NH}) \mathrm{H}-$ bond is lost. It has also to be noted that, in $\operatorname{Trp}_{\text {in }}$ simulations, Gln63 only stabilized in the final conformation after at least $200 \mathrm{~ns}$ of simulation, suggesting that the nanosecond MDs employed in previous works ${ }^{29,32,33}$ are not sufficient to equilibrate the $\operatorname{Trp}_{\text {in }}$ structure.

Our results also pose a challenge to some model mechanisms used to explain the dark-to-light conversion in AppA, ${ }^{20,23}$ which were based on the $\operatorname{Trp}_{\text {in }}$ crystal structure. ${ }^{16}$ In fact, a structure with the Gln63 oxygen oriented towards Trp104 would be highly unstable, and would relax in less than a $\mu$ s towards the opposite Gln63 orientation. Such a structure is obviously incompatible with either the dark-adapted or the light-induced state. It is important to stress that X-ray crystallography cannot distinguish the two orientations of Gln63, and in the crystal structure of $\operatorname{Trp}_{\text {in }}$ (PDB: 1YRX) the orientation with the amide oxygen towards Trp104 was chosen only 
on the basis of a possible hydrogen bond with the pyrrolic $\mathrm{NH}$ group ${ }^{16}$. However, our results demonstrate that this hydrogen bond is not stable, and in turn suggest that the Gln63 orientation present in the $\operatorname{Trp}_{\text {in }}$ crystal is an artifact. Notably, it was also argued that the orientation chosen by Anderson et al. does not fit well the X-ray electron density. ${ }^{63}$

In contrast to Gln63, Met106 and Trp104 are both stable when they are inside the flavin binding pocket. Therefore, both $\operatorname{Trp}_{i n}$ and Met $_{i n}$ are possible candidates for the dark-adapted structure of AppA, or, similarly, for the light-induced state. Our results also confirm that Trp104 is stable inside the pocket even when the Gln63 amide nitrogen is close to the Trp-NH group, in contrast with previous speculations. ${ }^{34}$ Our MDs suggest that the Met $_{\text {in }}$ structure is more stable than the Trp $\mathrm{T}_{\text {in }}$ one, especially in the binding pocket, which is more compact and exhibits stronger hydrogen bonds. However, when the mutation of $\mathrm{Met}_{\text {in }}$ is reverted back to the WT ana$\log , \mathrm{Met}_{i n}-\mathrm{S} 20 \mathrm{C}$, our simulations show larger instabilities and larger fluctuations in the core of the protein, similar to the original $\operatorname{Trp}_{i n}$ simulations. These results suggest that the C20S mutation can actively stabilize the Met $_{i n}$ structure, possibly at the expense of $\operatorname{Trp}_{i n}$. It would be therefore important to have an experimental demonstra-

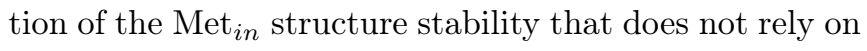
amino acid mutations.

The integration of MD analysis with an investigation of NMR chemical shifts has revealed further important aspects. According to our results, the $\operatorname{Trp}_{i n}$ and Met ${ }_{i n}$ structures give very similar chemical shifts for the flavin protons. The $\mathrm{H}_{3}$ proton, which is a reporter of the lightinduced state,${ }^{20}$ did not present statistically significant chemical shift differences between Met ${ }_{i n}$ and $\operatorname{Trp}_{i n}$. Only one amide proton of Asn45 gave strikingly different results for $\operatorname{Met}_{i n}$ and $\operatorname{Trp}_{i n}$. In particular, the chemical shift calculated for the $\operatorname{Trp}_{\text {in }}$ structure is much lower than the experiment. We traced back this unusual chemical shift to a strong shielding effect of the aromatic Trp104 ring, which is located close to Asn45 in the Trp ${ }_{\text {in }}$ structure. Our NMR results indicate that the Trpin structure is not compatible with the experiments, thus challening the position and orientation of Trp104 in the Trp in $_{\text {in }}$ structure. A different orientation of tryptophan was detected inside the binding pocket of another protein of the BLUF family (Slr1694). ${ }^{64}$ In this structure, the indole ring of the tryptophan is flipped with its nitrogen atom pointing away from the pocket. A detailed computational study by Hammes-Schiffer and co-workers ${ }^{65}$ examined the stability and the effect of the latter conformation of the tryptophan on the energy of Slr1694 protein and compared them to those of the conformation adopted in AppA by changing the orientation of the tryptophan manually in Slr1694. The authors concluded that the conformation of the tryptophan pointing away from the pocket is thermodynamically more stable than the conformation found in AppA. Our NMR simulations seems to suggest that this finding can also be valid for AppA.

Finally, $\operatorname{Trp}_{i n}$ and $\mathrm{Met}_{\text {in }}$ configurations have been tested within the context of the two spectroscopic signatures used to characterize the dark-to-light conversion, the redshifts in the frequency of the $\mathrm{C}_{4}=\mathrm{O}_{4}$ stretching mode and in the UV-Vis absorption maximum. Both analyses show much weaker $\mathrm{H}$-bonding interactions within the binding pocket for the structures resulting from the $\operatorname{Trp}_{\text {in }}$ trajectories, which do not allow to identify any significant correlation between the investigated spectroscopic properties and specific arrangement of the residues around the flavin. Instead, a more predictable picture comes out for $\mathrm{Met}_{\text {in }}$ configurations, for which we found a clear correlation between the frequency of the $\mathrm{C}_{4}=\mathrm{O}_{4}$ stretching mode and the $\mathrm{O}_{4}-\mathrm{Gln} 63(\mathrm{H})$ distance. The analysis of the vibrational frequencies, however, has also shown that the dynamics and the relative weakness of the intermolecular interactions within the binding pocket requires to go beyond an harmonic model to achieve a realistic description. Finally, our results have shown that the analysis of excitation energies is very delicate as their values depend more strongly on small distortions of the flavin geometry rather than on the direct effects of nearby residues on the relative energies of the electronic states.

Several studies have suggested that dark-to-light spectral differences could be explained by keto-enol tautomerization of Gln63, possibly also involving a flipping of the side chain. ${ }^{22,30,63,66}$ This mechanism could explain the spectral differences between dark and light states of $\mathrm{AppA}^{22}$ or Slr1694, ${ }^{67}$ better than the $\operatorname{Trp}_{\text {in }} /$ Met $_{\text {in }}$ differences. Our simulations, however, suggest that both the spectral features and the orientation of Gln63 are insensitive to whether Trp104 or Met106 are present inside the pocket. This result should be taken into account when considering the tautomerization mechanism of BLUF photoactivation. 


\section{Conclusions}

In this work we have shown that atomistic simulations can nowadays represent a feasible and reliable strategy to characterize the structure of photoreceptors in their natural environment. Such a result has been made possible thanks to the combination of microsecond molecular dynamics with accurate multiscale calculations of very different spectroscopies.

Here, this integrated approach has been used to uncover the structure of the dark state of AppA BLUF photoreceptor in solution and to investigate some of the most popular molecular mechanisms present in the literature for the dark-to-light activation. Only the combination of NMR, IR and UV-Vis simulated spectroscopies has allowed to reach a robust structural picture of the dark state showing, for example, that the Gln63 side chain presents a unique a definite preferential orientation of irrespective of whether Trp104 is inside or outside the flavin-binding pocket, and that Trp104 should present another conformation inside the pocket. These findings finally challenge the popular model of AppA BLUF activation based on Gln flipping and the Met106/Trp104 substitution inside the binding pocket, and show that the dark-to-light transition cannot be explained by these structural changes.

\section{Acknowledgements}

The authors acknowledge funding by the European Research Council, under the grant ERC-AdG-786714 (LIFETimeS).

\section{References}

[1] J. M. Thornton, A. E. Todd, D. Milburn, N. Borkakoti and C. A. Orengo, Nat. Struct. Biol., 2000, 7 Suppl, 991-994.

[2] M. A. van der Horst and K. J. Hellingwerf, Acc. Chem. Res., 2004, 37, 13-20.

[3] A. Möglich, X. Yang, R. A. Ayers and K. Moffat, Annu. Rev. Plant Biol., 2010, 61, 21-47.

[4] T. Kottke, A. Xie, D. S. Larsen and W. D. Hoff, Annu. Rev. Biophys., 2018, 47, 291-313.

[5] E. Brini, C. Simmerling and K. Dill, Sci., 2020, 370, eaaz3041-10.
[6] H. M. Senn and W. Thiel, Angew. Chem. Int. Ed., 2009, 48, 1198-1229.

[7] M. Bondanza, M. Nottoli, L. Cupellini, F. Lipparini and B. Mennucci, Phys. Chem. Chem. Phys., 2020, 22, 14433-14448.

[8] S. Masuda, K. Hasegawa, A. Ishii and T. aki Ono, Biochemistry, 2004, 43, 5304-5313.

[9] S. Masuda, K. Hasegawa and T. A. Ono, Plant Cell Physiol., 2005, 46, 1894-1901.

[10] M. Unno, R. Sano, S. Masuda, T. aki Ono and S. Yamauchi, J. Phys. Chem. B, 2005, 109, 12620-12626.

[11] A. L. Stelling, K. L. Ronayne, J. Nappa, P. J. Tonge and S. R. Meech, J. Am. Chem. Soc., 2007, 129, $15556-15564$.

[12] S. Y. Park and J. R. Tame, Biophys. Rev., 2017, 9, 169-176.

[13] T. Fujisawa and S. Masuda, Biophys. Rev., 2018, 10, 327-337.

[14] T. Iwata, T. Nagai, S. Ito, S. Osoegawa, M. Iseki, M. Watanabe, M. Unno, S. Kitagawa and H. Kandori, J. Am. Chem. Soc., 2018, 140, 11982-11991.

[15] S. Masuda and C. E. Bauer, Cell, 2002, 110, 613623.

[16] S. Anderson, V. Dragnea, S. Masuda, J. Ybe, K. Moffat and C. Bauer, Biochemistry, 2005, 44, 7998-8005.

[17] A. Jung, J. Reinstein, T. Domratcheva, R. L. Shoeman and I. Schlichting, J. Mol. Biol., 2006, 362, 717-732.

[18] M. Unno, S. Masuda, T.-a. Ono and S. Yamauchi, J. Am. Chem. Soc., 2006, 128, 5638-5639.

[19] S. Masuda, Y. Tomida, H. Ohta and K. ichiro Takamiya, J. Mol. Biol., 2007, 368, 1223-1230.

[20] J. S. Grinstead, M. Avila-Perez, K. J. Hellingwerf, R. Boelens and R. Kaptein, J. Am. Chem. Soc., 2006, 128, 15066-15067.

[21] M. Gauden, S. Yeremenko, W. Laan, I. H. Van Stokkum, J. A. Ihalainen, R. Van Grondelle, K. J. Hellingwerf and J. T. Kennis, Biochemistry, 2005, 44, 3653-3662. 
[22] F. Collette, T. Renger and M. Schmidt am Busch, J. Phys. Chem. B, 2014, 118, 11109-11119.

[23] P. Goyal and S. Hammes-Schiffer, Proc. Natl. Acad. Sci. U. S. A., 2017, 114, 1480-1485.

[24] M. G. Khrenova, A. V. Nemukhin and T. Domratcheva, J. Phys. Chem. B, 2013, 117, 2369-2377.

[25] T. Domratcheva, E. Hartmann, I. Schlichting and T. Kottke, Sci. Rep., 2016, 6, 1-14.

[26] V. Dragnea, A. I. Arunkumar, H. Yuan, D. P. Giedroc and C. E. Bauer, Biochemistry, 2009, 48, 9969-9979.

[27] R. Brust, A. Lukacs, A. Haigney, K. Addison, A. Gil, M. Towrie, I. P. Clark, G. M. Greetham, P. J. Tonge and S. R. Meech, J. Am. Chem. Soc., 2013, 135, 16168-16174.

[28] K. Karadi, S. M. Kapetanaki, K. Raics, I. Pecsi, R. Kapronczai, Z. Fekete, J. N. Iuliano, J. T. Collado, A. A. Gil, J. Orban, M. Nyitrai, G. M. Greetham, M. H. Vos, P. J. Tonge, S. R. Meech and A. Lukacs, Sci. Rep., 2020, 10, 1-15.

[29] K. Obanayama, H. Kobayashi, K. Fukushima and M. Sakurai, Photochem. Photobiol., 2008, 84, 10031010.

[30] M. G. Khrenova, A. V. Nemukhin, B. L. Grigorenko, A. I. Krylov and T. M. Domratcheva, J. Chem. Theory Comput., 2010, 6, 2293-2302.

[31] Y.-W. Hsiao, J. P. Götze and W. Thiel, J. Phys. Chem. B, 2012, 116, 8064-8073.

[32] J. P. Götze, C. Greco, R. Mitrić, V. BonačićKoutecký and P. Saalfrank, J. Comput. Chem., 2012, 33, 2233-2242.

[33] K. Meier, W. Thiel and W. F. Van Gunsteren, J. Comput. Chem., 2012, 33, 363-378.

[34] T. Mathes and J. P. Götze, Front. Mol. Biosciences, 2015, 2, 1-14.

[35] R. Anandakrishnan, B. Aguilar and A. V. Onufriev, Nucleic Acids Res., 2012, 40, W537-W541.

[36] J. A. Maier, C. Martinez, K. Kasavajhala, L. Wickstrom, K. E. Hauser and C. Simmerling, J. Chem. Theory Comput., 2015, 11, 3696-3713.
[37] D. A. Case, I. Y. Ben-Shalom, S. R. Brozell, D. S. Cerutti, T. E. Cheatham, III, V. W. D. Cruzeiro, T. A. Darden, R. Duke, D. Ghoreishi, M. K. Gilson, H. Gohlke, A. W. Goetz, D. Greene, R. Harris, N. Homeyer, S. Izadi, A. Kovalenko, T. Kurtzman, T. S. Lee, S. LeGrand, P. Li, C. Lin, J. Liu, T. Luchko, R. Luo, D. J. Mermelstein, K. M. Merz, Y. Miao, G. Monard, C. Nguyen, H. Nguyen, I. Omelyan, A. Onufriev, F. Pan, R. Qi, D. R. Roe, A. Roitberg, C. Sagui, S. Schott-Verdugo, J. Shen, C. L. Simmerling, J. Smith, R. Salomon-Ferrer, J. Swails, R. C. Walker, J. Wang, H. Wei, R. M. Wolf, X. Wu, L. Xiao, D. M. York and P. A. Kollman, AMBER 2018, 2018, University of California, San Francisco.

[38] T.-S. Lee, D. S. Cerutti, D. Mermelstein, C. Lin, S. LeGrand, T. J. Giese, A. Roitberg, D. A. Case, R. C. Walker and D. M. York, J. Chem. Inf. Model., 2018, 58, 2043-2050.

[39] J. Wang, R. M. Wolf, J. W. Caldwell, P. A. Kollman and D. A. Case, J. Comput. Chem., 2004, 25, 11571174 .

[40] C. Schneider and J. Suhnel, Biopolymers, 1999, 50, 287-302.

[41] L. W. Chung, W. M. C. Sameera, R. Ramozzi, A. J. Page, M. Hatanaka, G. P. Petrova, T. V. Harris, X. Li, Z. Ke, F. Liu, H.-B. Li, L. Ding and K. Morokuma, Chem. Rev., 2015, 115, 5678-5796.

[42] S. Grimme, J. Antony, S. Ehrlich and H. Krieg, J. Chem. Phys., 2010, 132, 154104.

[43] C. Curutchet, A. Muñoz Losa, S. Monti, J. Kongsted, G. D. Scholes and B. Mennucci, J. Chem. Theory Comput., 2009, 5, 1838-1848.

[44] F. Lipparini, J. Chem. Theory Comput., 2019, 15, $4312-4317$.

[45] J. Wang, P. Cieplak, J. Li, T. Hou, R. Luo and Y. Duan, J. Phys. Chem. B, 2011, 115, 3091-9.

[46] J. Wang, P. Cieplak, J. Li, J. Wang, Q. Cai, M. Hsieh, H. Lei, R. Luo and Y. Duan, J. Phys. Chem. B, 2011, 115, 3100-3111.

[47] F. Cardoso Ramos, L. Cupellini and B. Mennucci, J. Phys. Chem. B, 2021, 125, 1768-1777. 
[48] S. Mukamel, Principles of Nonlinear Optical Spectroscopy, Oxford University Press, New York, 1995.

[49] S. Hashem, L. Cupellini, F. Lipparini and B. Mennucci, Mol. Phys., 2020, 118, e1771449.

[50] D. Loco, L. Lagardère, S. Caprasecca, F. Lipparini, B. Mennucci and J.-P. Piquemal, J. Chem. Theory Comput., 2017, 13, 4025-4033.

[51] M. Nottoli, B. Mennucci and F. Lipparini, Phys. Chem. Chem. Phys., 2020, 22, 19532-19541.

[52] M. Nottoli, M. Bondanza, F. Lipparini and B. Mennucci, J. Chem. Phys., 2021, 154, 184107.

[53] J. A. Rackers, Z. Wang, C. Lu, M. L. Laury, L. Lagardère, M. J. Schnieders, J.-P. Piquemal, P. Ren and J. W. Ponder, J. Chem. Theory Comput., 2018, 14, 5273-5289.

[54] L. Lagardère, L.-H. Jolly, F. Lipparini, F. Aviat, B. Stamm, Z. F. Jing, M. Harger, H. Torabifard, G. A. Cisneros, M. J. Schnieders, N. Gresh, Y. Maday, P. Y. Ren, J. W. Ponder and J.-P. Piquemal, Chem. Sci., 2018, 9, 956-972.

[55] M. J. Frisch, G. W. Trucks, H. B. Schlegel, G. E. S. andM. A. Robb, J. R. Cheeseman, G. Scalmani, V. B. andG. A. Petersson, H. Nakatsuji, X. Li, A. V. Marenich, M. C. andJ. Bloino, B. G. Janesko, J. Zheng, R. Gomperts, B. M. andH. P. Hratchian, J. V. Ortiz, A. F. Izmaylov, J. L. S. andD. WilliamsYoung, F. Ding, F. Lipparini, F. Egidi, J. G. andB. Peng, A. Petrone, T. Henderson, D. Ranasinghe, V. G. Z. andJ. Gao, N. Rega, G. Zheng, W. Liang, M. Hada, M. Ehara, K. T. andR. Fukuda, J. Hasegawa, M. Ishida, T. Nakajima, Y. Honda, O. K. andH. Nakai, T. Vreven, K. Throssell, J. a. E. P. J. A. Montgomery, F. Ogliaro, M. J. Bearpark, J. J. H. andE. N. Brothers, K. N. Kudin, V. N. Staroverov, T. A. K. andR. Kobayashi, J. Normand, K. Raghavachari, A. P. R. andJ. C. Burant, S. S. Iyengar, J. Tomasi, M. Cossi, J. M. M. andM. Klene, C. Adamo, R. Cammi, J. W. Ochterski, R. L. M. andK. Morokuma, O. Farkas, J. B. Foresman, and D. J. Fox, Gaussian Development Version, Revision J.13, 2020, Gaussian, Inc., Wallingford CT, 2020.

[56] P. Ren and J. W. Ponder, J. Phys. Chem. B, 2003, 107, 5933-5947.
[57] J. W. Ponder, C. Wu, P. Ren, V. S. Pande, J. D. Chodera, M. J. Schnieders, I. Haque, D. L. Mobley, D. S. Lambrecht, R. A. DiStasio, M. Head-Gordon, G. N. I. Clark, M. E. Johnson and T. Head-Gordon, J. Phys. Chem. B, 2010, 114, 2549-2564.

[58] A. Belouchrani, K. Abed-Meraim, J.-F. Cardoso and E. Moulines, Ieee Trans. Signal Process., 1997, 45, 434-444.

[59] J. S. Grinstead, S. T. D. Hsu, W. Laan, A. M. Bonvin, K. J. Hellingwerf, R. Boelens and R. Kaptein, Chembiochem, 2006, 7, 187-193.

[60] Q. Wu, W. H. Ko and K. H. Gardner, Biochemistry, 2008, 47, 10271-10280.

[61] B. J. Kraft, S. Masuda, J. Kikuchi, V. Dragnea, G. Tollin, J. M. Zaleski and C. E. Bauer, Biochemistry, 2003, 42, 6726-6734.

[62] A. Haigney, A. Lukacs, R. Brust, R.-K. Zhao, M. Towrie, G. M. Greetham, I. Clark, B. Illarionov, A. Bacher, R.-R. Kim, M. Fischer, S. R. Meech and P. J. Tonge, J. Phys. Chem. B, 2012, 116, 1072210729 .

[63] A. Udvarhelyi and T. Domratcheva, J. Phys. Chem. B, 2013, 117, 2888-2897.

[64] H. Yuan, S. Anderson, S. Masuda, V. Dragnea, K. Moffat and C. Bauer, Biochemistry, 2006, 45, 12687-12694.

[65] J. J. Goings, C. R. Reinhardt and S. HammesSchiffer, J. Am. Chem. Soc., 2018, 140, 1524115251.

[66] T. Domratcheva, B. Grigorenko, I. Schlichting and A. Nemukhin, Biophys. J., 2008, 94, 3872-3879.

[67] J. J. Goings, P. Li, Q. Zhu and S. Hammes-Schiffer, Proc. Natl. Acad. Sci., 2020, 117, 26626-26632. 